\title{
Guía de densidad para el manejo de rodales naturales de Pinus rudis Endl. en Oaxaca
}

\section{Density management guide for natural stands of Pinus rudis Endl. in Oaxaca}

\author{
Daniel Martínez Santiago ${ }^{1 *}$, Prudencia Caballero Cruz ${ }^{2}$, Eduardo Filio Hernández ${ }^{1}$, \\ Alejandro Garzón Trinidad ${ }^{1}$, Rosalino Ortiz Barrios ${ }^{1}$, Octavio Lemuel Cruz Santiago ${ }^{1}$, \\ Ithaiz Aparicio Cuevas ${ }^{1}$ y Carmela Sandoval García ${ }^{1}$
}

\begin{abstract}
Forest density associated with site quality determines forest productivity and its measurement allows prediction of growth and mortality rates. Management through thinning improves forest characteristics by reducing competition between trees. The aim of the present study was to construct a density guide for the management of natural stands of Pinus rudis, in the Mixteca region of Oaxaca, Mexico. The Reineke's Stand Density Index (SDI) was estimated from the quadratic diameters and number of trees per hectare of 81 variable size sampling sites, located in pure forest, with full occupation and different development stages of forest stands. The Crown Competition Factor (CCF) was estimated by fitting the simple linear regression model with normal diameter-crown diameter data from 54 healthy trees, without physical-mechanical defects and that grown without competition. The adjustment of the Reineke model and that of simple linear regression was carried out using the ordinary least squares technique. The density guide was developed through SDI and CCF, these measurements allowed to know that the stands under study have density levels higher than $90 \%$ of the SDI, with a quadratic diameter of $15 \mathrm{~cm}$.
\end{abstract}

Key words: Thinning, competition, crown competition, Reineke's density index, forest management, forestry.

\section{Resumen}

La densidad forestal asociada con la calidad de sitio determina la productividad del bosque, y su medición permite predecir tasas de crecimiento y mortalidad. El manejo a través de aclareos mejora las características del bosque, ya que reduce la competencia entre individuos. El propósito del presente estudio, fue construir una guía de densidad para el manejo de rodales naturales de Pinus rudis en la región Mixteca de Oaxaca, México. El índice de densidad de Reineke (IDR) se calculó a partir de los diámetros cuadráticos y número de árboles por hectárea de 81 sitios de muestreo de dimensiones variables, ubicados en masas puras, de ocupación completa y diferentes etapas de crecimiento. El factor de competencia de copas (FCC) se estimó mediante el ajuste del modelo de regresión lineal simple, con datos del diámetro normal-diámetro de copa de 54 árboles sin presencia de plagas o enfermedades, defectos físico-mecánicos y que estaban creciendo libres de competencia. El ajuste del modelo de Reineke y el de regresión lineal simple se realizó con la técnica de Mínimos Cuadrados Ordinarios. A partir del IDR y el FCC se construyó una guía de densidad, con la cual se determinó que los rodales objeto de estudio presentan niveles de densidad por arriba de $90 \%$ de IDR, con un diámetro cuadrático de $15 \mathrm{~cm}$.

Palabras clave: Aclareos, competencia, factor de competencia de copas, índice de densidad de Reineke, manejo forestal, silvicultura.

Fecha de recepción/Reception date: 22 de agosto de 2020

Fecha de aceptación/Acceptance date: 10 de diciembre de 2020

${ }^{1}$ Tecnológico Nacional de México/Instituto Tecnológico Superior de San Miguel el Grande. México.

${ }^{2}$ Centro de Investigación, Divulgación, Asesonía Técnica Forestal y Agropecuaria S. C. México.

*Autor por correspondencia; correo-e: masd8510@gmail.com 


\section{Introducción}

Las guías o diagramas para el manejo de la densidad son una herramienta de importancia para la gestión forestal, ya que sirven como instrumento para definir el número óptimo de individuos que crecen en un rodal a diferentes etapas de desarrollo (Navarro et al., 2011). Además, con ellas se puede aumentar la productividad maderable a través de una buena planificación y monitoreo de las intervenciones silvícolas (Gezan et al., 2007; Hernández et al., 2013).

Existen métodos absolutos y relativos para evaluar la densidad de un rodal o plantación; los primeros hacen referencia al número de árboles y al área basal por hectárea y proporcionan una estimación del número de individuos presentes en una población; sin embargo, no dan información acerca de la competencia en el sitio (Torres y Velázquez, 2000). Los relativos, como los índices de Densidad de Reineke (IDR) (Reineke, 1933), el de Yoda (IDY) (Yoda et al., 1963), relación área-árbol (RAA) (Chisman y Schumacher, 1940), factor de competencia de copas (FCC) (Krajicek et al., 1961) y el de densidad relativa (Curtis, 1970) describen el nivel de densidad del rodal y sirven como indicadores de las necesidades de aplicación de tratamientos silvícolas en el bosque (Torres y Velázquez 2000; Torres y Magaña, 2001).

El IDR es una de las medidas de densidad relativa más utilizada en la construcción de guías o diagramas de densidad (Tamarit et al., 2020); se basa en la relación funcional que existe entre el número de árboles por hectárea de un rodal y su diámetro cuadrático (Quiñonez et al., 2017; Tamarit et al., 2020). A partir de este, se han generado líneas de autoaclareo para bosques de coníferas, latifoliadas y con mezcla de especies (Gezan et al., 2007; Navarro et al., 2011; Santiago et al., 2013; Quiñonez et al., 2017; Tamarit et al., 2018).

La primera guía de densidad la desarrolló Gingrich (1967) para taxones de hojosas en los estados centrales de Estados Unidos de América. En México, su generación data de los años 80 del siglo pasado, con autores como Zepeda y Villareal (1987), quienes elaboraron una para Pinus hartwegii Lindl. en el centro del país. Márquez y Álvarez (1995) construyeron una guía para el manejo de rodales de Pinus cooperi var. ornelasi (Martínez) Blanco, en 
Durango; y García et al. (1996) una para el manejo de plantaciones de Swietenia macrophylla King en el estado de Quintana Roo. En los casos anteriores, los índices de densidad utilizados fueron RAA, FCC e IDR.

Santiago et al. (2013) y Tamarit et al. (2018) construyeron diagramas de densidad para Pinus patula Schiede ex Schltdl. \& Cham. en los estados de Hidalgo y Puebla, respectivamente; Quiñonez et al. (2017) lo hicieron para bosques mezclados del norte de México. El desarrollo de esas herramientas ha sido, principalmente, para la prescripción de aclareos con propósitos de producción maderable.

Pinus rudis Endl. es una especie que se distribuye ampliamente en la república mexicana, en un intervalo altitudinal 2200 a 3300 m; aunque es posible encontrarla por arriba de los 3300 msnm. Las alturas de sus individuos varían de 20 a 30 m, con diámetros de 40 a $70 \mathrm{~cm}$. Se asocia con Pinus ayacahuite Ehrenb. ex Schltdl, $P$. montezumae Lamb y $P$. hartwegii. Su madera se destina, sobre todo, para la construcción (Perry, 1991).

Entre los años 1998 y 2005 diversas poblaciones de $P$. rudis fueron afectados por incendios forestales en la comunidad agraria de San Miguel El Grande, región Mixteca de Oaxaca (Martínez, 2009). En consecuencia, esas áreas tienen alta densidad de regeneración natural posincendio (Caballero et al., 2018). En este contexto, el objetivo del presente estudio fue construir una guía de densidad para el manejo de rodales naturales de Pinus rudis, en la región Mixteca de Oaxaca, México. 


\section{Materiales y Métodos}

\section{Área de estudio}

La comunidad agraria de San Miguel El Grande se ubica al oeste de Oaxaca, a una altitud que fluctúa de 2200 a 3330 m, entre las coordenadas extremas 1658'29" y 17010'27" latitud norte, 97³3'23" y 97040'21" longitud oeste. El estudio se realizó en el área de distribución natural de $P$. rudis, en una superficie aproximada de 1242 ha (Figura 1). Los tipos de climas predominantes en la comunidad son $\mathrm{Cb}^{\prime}\left(\mathrm{w}_{2}\right)$ y $\mathrm{C}\left(\mathrm{c}_{2}\right)$, con lluvias en verano (García, 1998). Los suelos corresponden a los tipos regosoles, litosoles y vertisoles (INEGI, 2014). La vegetación está representada por bosques de pino y pino-encino (INEGI, 2016), en donde las principales especies de coníferas son Pinus oaxacana Mirov, P. pseudostrobus Lindl., P. douglasiana Martínez, P. rudis, P. oocarpa Schiede y P. leiophylla Schltdl. et Cham. y Abies hickelii Flous \& Gaussen; en menor proporción crecen taxones de los géneros Quercus y Arbutus (Martínez, 2009).

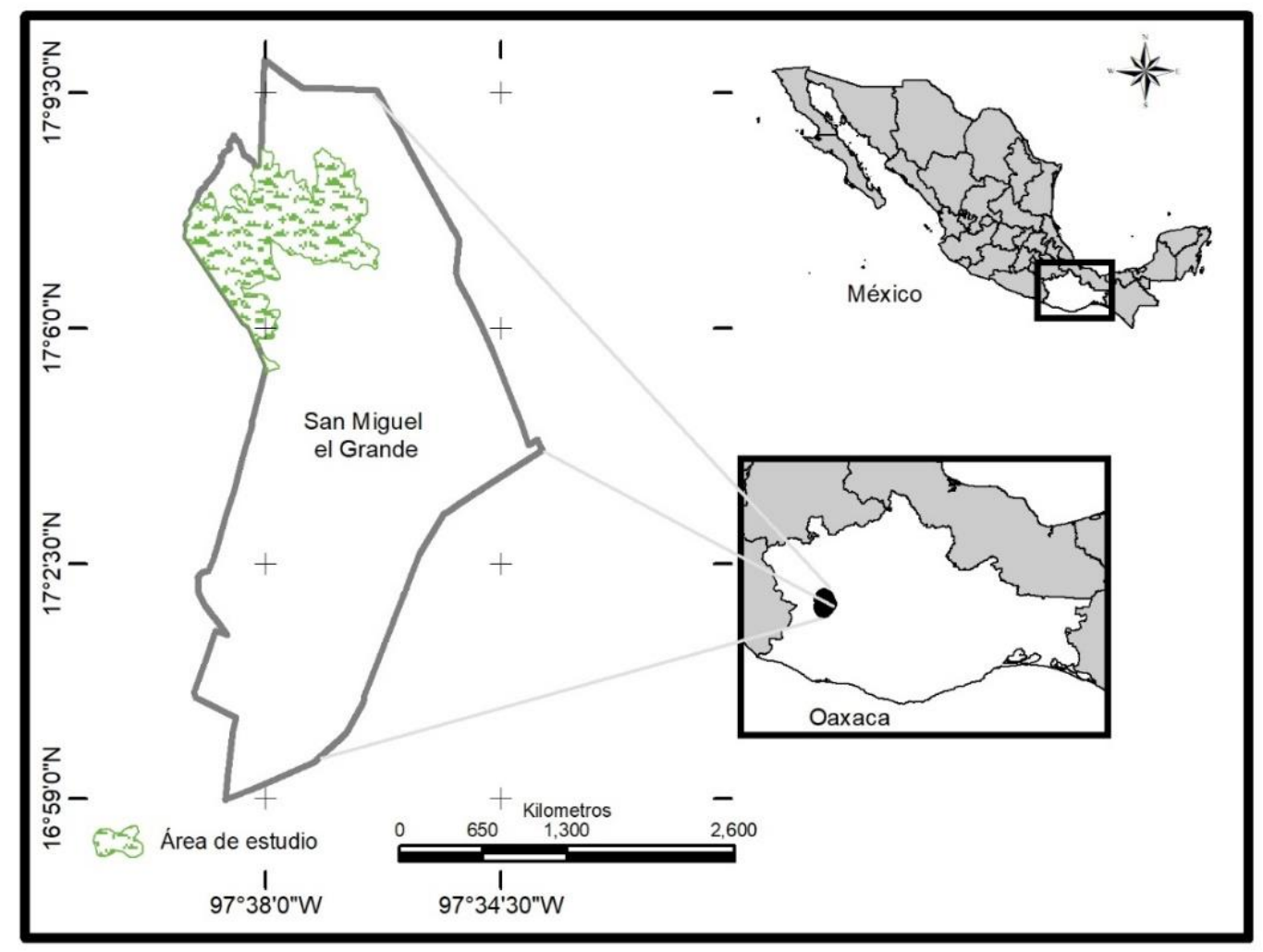

Figura 1. Localización geográfica del área de estudio. 


\section{Estimación del índice de densidad de Reineke}

Para la estimación de este índice se ubicaron, de manera selectiva, 81 sitios de dimensiones variables; para ello, se utilizó el método de muestreo propuesto por Prodan (1968), el cual considera la medición de seis árboles, cuya unidad de muestreo queda definida del centro del sitio al centro del sexto árbol (Figura 2). Para la selección del sitio se consideraron áreas de bosque entre monte bravo y fustal (Müller et al., 2013; Aguilar, 2018), masas con presencia de la misma especie en más de $90 \%$, áreas homogéneas de ocupación completa, sitios con arbolado sano y sin daños físicos (Lee y Choi, 2019).

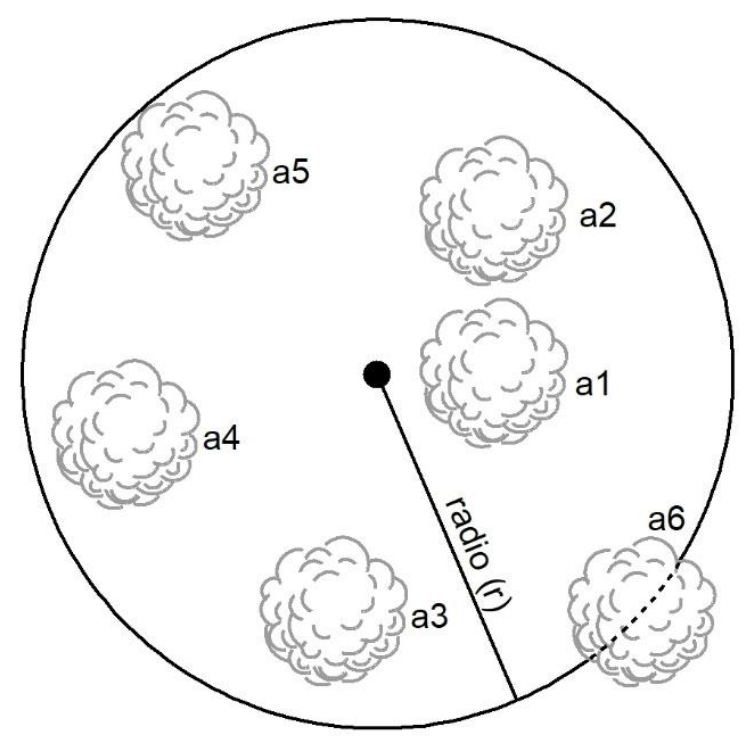

Figura 2. Sitio de muestreo de seis árboles.

A todos los individuos del sitio de muestreo se les midió el diámetro normal $(D)$ con cinta diamétrica (Forestry Suppliers, modelo $283 \mathrm{~d} / 160 \mathrm{~cm}$ ), a $1.3 \mathrm{~m}$ de la altura del árbol, con esta variable se estimó el área basal de los individuos presentes dentro del sitio de muestreo. 


$$
g=\frac{\pi}{4}\left(\frac{D}{100}\right)^{2}
$$

Donde:

$g=$ Área basal individual $\left(\mathrm{m}^{2}\right)$

$D=$ Diámetro normal $(\mathrm{cm})$

$\Pi / 4=$ Constante $(0.7854)$

Se estimaron dos medidas de densidad absoluta: área basal por hectárea $(G)$ y número de árboles por hectárea $(N)$, se emplearon las ecuaciones descritas por Ramos et al. (2017).

$$
\begin{aligned}
& N=N_{i}\left(\frac{S h}{S e}\right) \\
& G=G_{i}\left(\frac{S h}{S e}\right)
\end{aligned}
$$

Donde:

$N=$ Número de individuos por hectárea

$N_{i}=$ Número de individuos por sitio

$G=$ Área basal por hectárea $\left(\mathrm{m}^{2}\right)$

$G_{i}=$ Área basal por sitio $\left(\mathrm{m}^{2}\right)$

$\mathrm{Sh}=$ Superficie de una hectárea $\left(\mathrm{m}^{2}\right)$

$\mathrm{Se}=$ Superficie evaluada en el sitio de muestreo $\left(\mathrm{m}^{2}\right)$

Estimados estos dos indicadores ( $G$ y $N$ ), se calculó el diámetro cuadrático $(D q)$ de cada parcela de muestreo, con la siguiente expresión (Santiago et al., 2013): 


$$
D q=\sqrt{\frac{40000}{\pi} \times \frac{A b}{N a}}
$$

Donde:

$D q=$ Diámetro cuadrático $(\mathrm{cm})$

$A b=$ Área basal por hectárea $\left(\mathrm{m}^{2}\right)$

$N a=$ Número de árboles por hectárea

$\Pi=$ Constante $(3.1416)$

Los valores por sitio de $D q$ y $N a$ se emplearon para el ajuste del modelo de Reineke (5), el cual definió la línea de densidad máxima promedio de la guía (línea A). Con fines de comparación de la densidad de rodales, en este índice se considera un $D q$ de $25 \mathrm{~cm}$ o 10 pulgadas (Reineke, 1933; Santiago et al., 2013; Hernández et al., 2013).

$$
N a=\beta_{0} D q^{\beta_{1}}
$$

Donde:

$N a=$ Número de árboles por hectárea

$D q=$ Diámetro cuadrático $(\mathrm{cm})$

$\beta_{0}=$ Intercepto al eje de las ordenadas

$\beta_{1}=$ Pendiente

El $A b$ fue producto del área basal individual a un diámetro dado, por el número de árboles estimados con la ecuación 5 (Rodríguez et al., 2009):

$$
A b=\frac{\pi}{4} \times\left(\frac{D}{100}\right)^{2} \times N a
$$


Donde:
$A b=$ Área basal por hectárea $\left(\mathrm{m}^{2}\right)$
$D=$ Diámetro normal $(\mathrm{cm})$
$N a=$ Número de árboles por hectárea
$\Pi / 4=$ Constante $(0.7854)$

\section{Estimación del factor de competencia de copas}

Esta medida de densidad se estimó a través de un muestreo dirigido a 54 árboles que crecieron libres de competencia, sanos y sin defectos físico-mecánico. Las variables evaluadas a cada individuo fueron diámetro $(D)$ y diámetro de copa $(D c)$, el cual se midió con un longímetro marca Pretul, modelo Pro-30me, dicha medición se realizó en dirección norte-sur y este-oeste, para obtener el promedio por individuo (Rodríguez et al., 2009).

Los 54 pares de datos $(D-D c)$, se emplearon para estimar el $D c$ en función al $D(7)$, una vez estimado, se calcularon las áreas de copa $(A C)$ (8) correspondientes a los árboles libres de competencia a un diámetro determinado (Rodríguez et al., 2009; Hernández et al., 2013).

$$
\begin{aligned}
& D C=\beta_{0}+\beta_{1} D \\
& A C=\frac{\pi}{4} \times D c^{2}
\end{aligned}
$$


Donde:

$D c=$ Diámetro de copa $(\mathrm{m})$

$D=$ Diámetro normal $(\mathrm{cm})$

$\beta_{0}=$ Intercepto al eje de las ordenadas

$\beta_{1}=$ Pendiente

$A c=$ Área de copa $\left(\mathrm{m}^{2}\right)$

$\Pi / 4=$ Constante $(0.7854)$

El área máxima de copa $(A m c)$ de un individuo, expresada como porcentaje de la unidad de superficie (ha) se determinó con la ecuación 9 (Rodríguez et al., 2009; Hernández et al., 2013):

$$
A m c=\frac{A c}{100}
$$

Donde:

$A m c=$ Área máxima de copa

$A C=$ Área de copa $\left(\mathrm{m}^{2}\right)$

Finalmente, el número de árboles y el área basal por hectárea se estimaron con las ecuaciones 10 y 11, respectivamente; con estos valores se calculó la línea de densidad suficiente de la guía (línea B) (Rodríguez et al., 2009; Hernández et al., 2013):

$$
\begin{gathered}
N a=\frac{100}{A m c} \\
A b=\frac{\pi}{4} \times\left(\frac{D}{100}\right)^{2} \times N a
\end{gathered}
$$


Donde:

$N a=$ Número de árboles por hectárea

$A m c=$ Área máxima de copa

$A b=$ Área basal por hectárea $\left(\mathrm{m}^{2}\right)$

$D=$ Diámetro normal $(\mathrm{cm})$

$\Pi / 4=$ Constante $(0.7854)$

\section{Ajuste de modelos}

El ajuste de las ecuaciones 5 y 7, se realizó por el método de Mínimos Cuadrados Ordinarios (MCO) en el software STATISTICA 10 (StatSoft Inc., 2011), a través de regresión no lineal y lineal, respectivamente. La calidad de ajuste de los modelos se evaluó mediante el valor del coeficiente de determinación $\left(r^{2}\right)$, la raíz del error medio cuadrático (REMC), así como el valor de su probabilidad (Vargas, 1999; Hernández et al., 2013).

\section{Construcción de la guía de densidad}

Para construir la guía de densidad, la línea A o de 100 \% (densidad máxima promedio) se definió con el $\mathrm{Na}$ ha-1 y $A b \mathrm{ha}^{-1}$ estimados a partir del modelo de Reineke, con estos valores se calcularon diferentes niveles de densidad (30 a $110 \%$ ) en intervalos de 10 $\%$. La línea B (densidad suficiente) fue determinada por el $N a \mathrm{ha}^{-1}$ y $A b \mathrm{ha}^{-1}$ resultado del FCC (Vargas, 1999). 


\section{Resultados y Discusión \\ Índice de densidad de Reineke}

El $D q$ explicó $94 \%\left(r^{2}=0.94\right)$ de la variación en el número de árboles por hectárea, con un error medio (REMC) de 831 y parámetros significativos a $95 \%$ de confiabilidad $(a<0.0001)$. La ecuación resultante fue la siguiente:

$$
N=149786.8 \times D q^{-1.55442}
$$

La representación gráfica de los valores observados ( $D q$ y $N a \cdot h a^{-1}$ ) mostró una dispersión de "J" invertida, la cual se exhibe la curva de ajuste del modelo de Reineke (Figura 3).

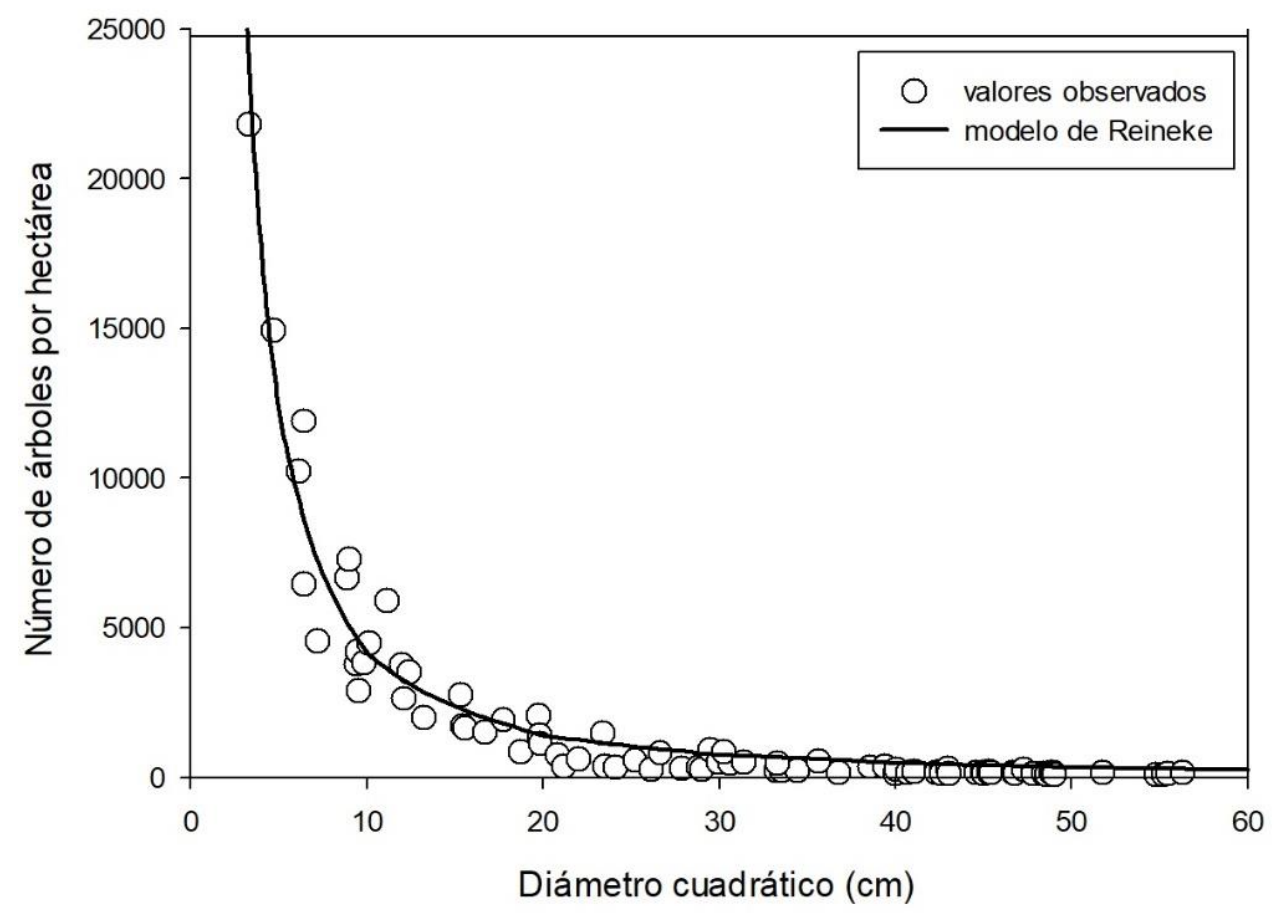

Figura 3. Relación entre el diámetro cuadrático y el número de árboles por hectárea. 
Los valores de las densidades máximas promedio, estimadas a partir del modelo de Reineke, fueron la base para construir el resto de las isolíneas de la guía de densidad (Cuadro 1).

Cuadro 1. Densidades máximas promedio estimadas con el modelo de Reineke.

\begin{tabular}{ccc}
\hline $\boldsymbol{q}$ & $\boldsymbol{N a} \mathbf{h a}^{-\mathbf{1}}$ & $\boldsymbol{A b} \mathbf{h a}^{-\mathbf{1}}$ \\
\hline 10 & 4179 & 32.82 \\
15 & 2225 & 39.32 \\
20 & 1423 & 44.70 \\
25 & 1006 & 49.37 \\
30 & 758 & 53.55 \\
35 & 596 & 57.35 \\
40 & 484 & 60.87 \\
45 & 403 & 64.15 \\
50 & 342 & 67.23 \\
\hline
\end{tabular}

$D q=$ Diámetro cuadrático $(\mathrm{cm}) ; N a \mathrm{ha}^{-1}=$ Número de árboles por hectárea; $A b \mathrm{ha}^{-1}=$ Área basal por hectárea.

Al considerar el valor del $D q$ de referencia $(25 \mathrm{~cm})$ se estimó un IDR de 1006 . En este sentido, Rodríguez et al. (2009) obtuvieron un valor de 1663 para $P$. montezumae Lamb; mientras que, Hernández et al. (2013) registraron un valor de 775 para Pinus teocote Schiede ex Schltdl. \& Cham., ambos estudios se realizaron en el estado de Hidalgo. Los valores aproximados de IDR solo son factibles de calcularse en rodales puros con densidad completa, de la misma especie y con el igual diámetro promedio del rodal (Reineke, 1933).

Se han estimado diversos valores de la pendiente $\left(\beta_{1}\right)$ para el modelo de Reineke, la mayoría varían entre -1.0151 (Rodríguez et al., 2009) y -2.18937 (Tamarit et al., 2018). Asimismo, se han desarrollado estudios para determinar, si el valor de $\beta_{1}$ postulado por Reineke (1993) es estadísticamente igual a -1.605; uno de ellos es el 
de Pretzsch y Biber (2005) quienes ajustaron la ecuación para bosques de Fagus sylvatica L., Picea abies (L.) Karst., Pinus sylvestris L. y Quercus petraea (Mattuschka) Liebl. en Alemania; excepto para P. sylvestris L., en el resto de los taxones el valor de la pendiente fue significativamente diferente.

Guezan et al. (2007) elaboraron diagramas de densidad para Nothofagus obliqua (Mirb.) Oerst, N. alpina (Poepp. \& Endl.) Oerst., N. dombeyi (Mirb.) Oerst. en Chile, y concluyeron que el valor del parámetro $\beta_{1}$ es específico para cada especie.

Santiago et al. (2013) construyeron guías de densidad para Pinus patula en el estado de Hidalgo, México. Las pendientes estimadas fueron de $-1.565 \pm 0.208$ para el modelo de Reineke y $1.199 \pm 0.048$ para el de Yoda. En el primer caso, se incluye al valor postulado por Reineke (1933). Asimismo, Quiñonez et al., (2017), en su trabajo de diagrama de densidad para bosques mezclados en Durango, México, obtienen un intervalo de -1.541 a -1.778 , el cual contiene el valor de -1.605 .

Santiago et al. (2013), Quiñonez et al. (2017) y Tamarit et al. (2018) compararon métodos de ajuste para definir la línea de autoaclareo, utilizaron Mínimos Cuadrados Ordinarios (MCO) y regresión frontera estocástica (RFE) y coinciden en que la RFE estima de forma eficiente el límite superior del autoaclareo. Por su parte, VanderSchaaf y Burkhart (2007) y Comeau et al. (2010) consideran que MCO también es un método apropiado para caracterizar la línea de densidad máxima.

En términos generales, el IDR se ha utilizado ampliamente en la construcción de diagramas o guías de densidad (Vargas, 1999; Gezan et al., 2007; Navarro et al., 2011; Santiago et al., 2013; Hernández et al., 2013; Quiñonez et al., 2017; Tamarit et al., 2018; Tamarit et al., 2020). Estos diagramas ofrecen mayor precisión en comparación a los construidos con el índice de Yoda y el de Espacio Relativo, ya que en el modelo de Reineke se usa el $D q$ calculado a partir del diámetro normal, el cual es una variable de medición directa; los otros dos utilizan el volumen y la altura total, respectivamente que se estiman, por lo general, con algún modelo matemático (Tamarit et al., 2020). 


\section{Factor de competencia de copas}

El diámetro $(D)$ explicó $83 \%\left(r^{2}=0.83\right)$ de la variación del $D c$, con un error medio (REMC) de 1.05 y parámetros significativos a $95 \%$ de confiabilidad $(a<0.0001)$. La ecuación resultante quedó definida como:

$$
D c=1.438794+0.1354 \times D
$$

En el Cuadro 2 se presentan los resultados obtenidos a partir de las ecuaciones 7-11, cuyo objetivo fue estimar el $N a h^{-1}$ y $A b h^{-1}$, para definir la línea de densidad suficiente de la guía.

Cuadro 2. Densidades estimadas a partir de factor de competencia de copas.

\begin{tabular}{cccccc}
\hline $\boldsymbol{D}$ & $\boldsymbol{D c}$ & $\boldsymbol{A c}$ & $\boldsymbol{A m c}$ & $\mathbf{N a ~ h a - 1}$ & $\boldsymbol{A b} \mathbf{h a}^{-1}$ \\
& $\mathbf{( 7 )}$ & $\mathbf{( 8 )}$ & $\mathbf{( 9 )}$ & $\mathbf{( 1 0 )}$ & $\mathbf{( 1 1 )}$ \\
\hline 10 & 2.79 & 6.13 & 0.061 & 1632 & 12.82 \\
15 & 3.47 & 9.46 & 0.095 & 1058 & 18.69 \\
20 & 4.15 & 13.51 & 0.135 & 740 & 23.26 \\
25 & 4.82 & 18.28 & 0.183 & 547 & 26.86 \\
30 & 5.50 & 23.77 & 0.238 & 421 & 29.74 \\
35 & 6.18 & 29.97 & 0.300 & 334 & 32.10 \\
40 & 6.85 & 36.90 & 0.369 & 271 & 34.05 \\
45 & 7.53 & 44.55 & 0.446 & 224 & 35.70 \\
50 & 8.21 & 52.92 & 0.529 & 189 & 37.10 \\
\hline
\end{tabular}

$D=$ Diámetro normal $(\mathrm{cm}) ; D c=$ Diámetro de copa $(\mathrm{m}) ; A c=$ Área de copa $\left(\mathrm{m}^{2}\right) ;$ $\mathrm{Na} h \mathrm{~h}^{-1}=$ Número de árboles por hectárea; $A b \mathrm{ha}^{-1}=$ Área basal por hectárea $\left(\mathrm{m}^{2}\right)$. 
Cualquier rodal que se encuentre por debajo del FCC (línea B), los árboles ahí presentes disponen de recursos suficientes para desarrollar todo su potencial de crecimiento, ya que no tienen competencia con otros individuos (Krajicek et al., 1961; Álvarez et al., 2004). Sin embargo, si se mantiene la densidad por debajo del FCC hasta su madurez, los árboles presentan mala calidad, si no se aplican podas (Krajicek et al., 1961). En este contexto, los individuos son resistentes a fuerzas mecánicas (viento y nieve), pero con desventajas en el rendimiento en aserrío, ya que tienen un bajo valor de esbeltez y un alto porcentaje de copa (Arias, 2005).

Cuando el rodal está sobre la línea B, se produce el cierre de copas. En este momento, comienza la competencia entre individuos; sin embargo, no se presenta una mortalidad inmediata (Gezan et al., 2007). Philbrook et al. (1973) señalan que el efecto de la competencia inicia cuando las existencias están a la mitad entre A y B; es decir, para este estudio por arriba de $70 \%$ del IDR.

Santiago et al. (2013) estimaron que la mortalidad natural ocurre en Pinus patula a partir de $55 \%$ del IDR. Quiñonez et al. (2017) definieron que en rodales con mezcla de especies, el efecto de la competencia se presenta cuando el valor del IDR es de $70 \%$.

\section{Construcción de la guía de densidad}

La línea A se definió con las densidades máximas promedio estimadas con el modelo de Reineke; los rodales que se encuentren por arriba de esta línea se consideran áreas sobrepobladas, las cuales necesitan una corta inmediata para mejorar la calidad del arbolado residual (Hernández et al., 2013; Santiago et al., 2013; Quiñonez et al., 2017; Tamarit et al., 2018).

La línea B se representó con el FCC, cualquier rodal que se ubique por debajo de esta línea se considera subpoblado o con densidad deficiente (Vargas, 1999; Rodríguez et al., 2009; Hernández et al., 2013). El rodal del presente estudio, requiere que se aumente su densidad para tomar decisiones respecto a su manejo, o en su caso, aplicar otro tratamiento intermedio 
para alcanzar el mejoramiento del sitio; por ejemplo, la fertilización si el objetivo central es la producción de madera (Daniel et al., 1982).

Para ejemplificar aplicación de la guía, se levantaron tres sitios de muestreo de $100 \mathrm{~m}^{2}(10 \times 10 \mathrm{~m})$ en un área de regeneración natural de Pinus rudis. A cada individuo se le midió el diámetro normal y se contabilizó el número de árboles por sitio, con estas variables se calculó el $A b \mathrm{ha}^{-1}$ y $\mathrm{Na}$ ha-1. Los valores promedio calculados fueron $38.27 \mathrm{~m}^{2}$ de $A b \mathrm{ha}^{-1}$ y $2045 \mathrm{Na}^{-1}$. Al ubicar estos valores en la guía (1), se estimó gráficamente un IDR de $96 \%$ y un $D q$ aproximado de $15.5 \mathrm{~cm}$.

Si se lleva al rodal de la condición 1 a la 2, se pasa a un nivel de densidad de $75 \%$; y se removerán $8.26 \mathrm{~m}^{2}$ de $A b \mathrm{ha}^{-1}$ y aproximadamente $545 \mathrm{Na} \mathrm{ha}^{-1}$. El propósito es mantener al rodal entre las líneas A y $B$ en una condición deseada de acuerdo a los objetivos de manejo (Figura 4).

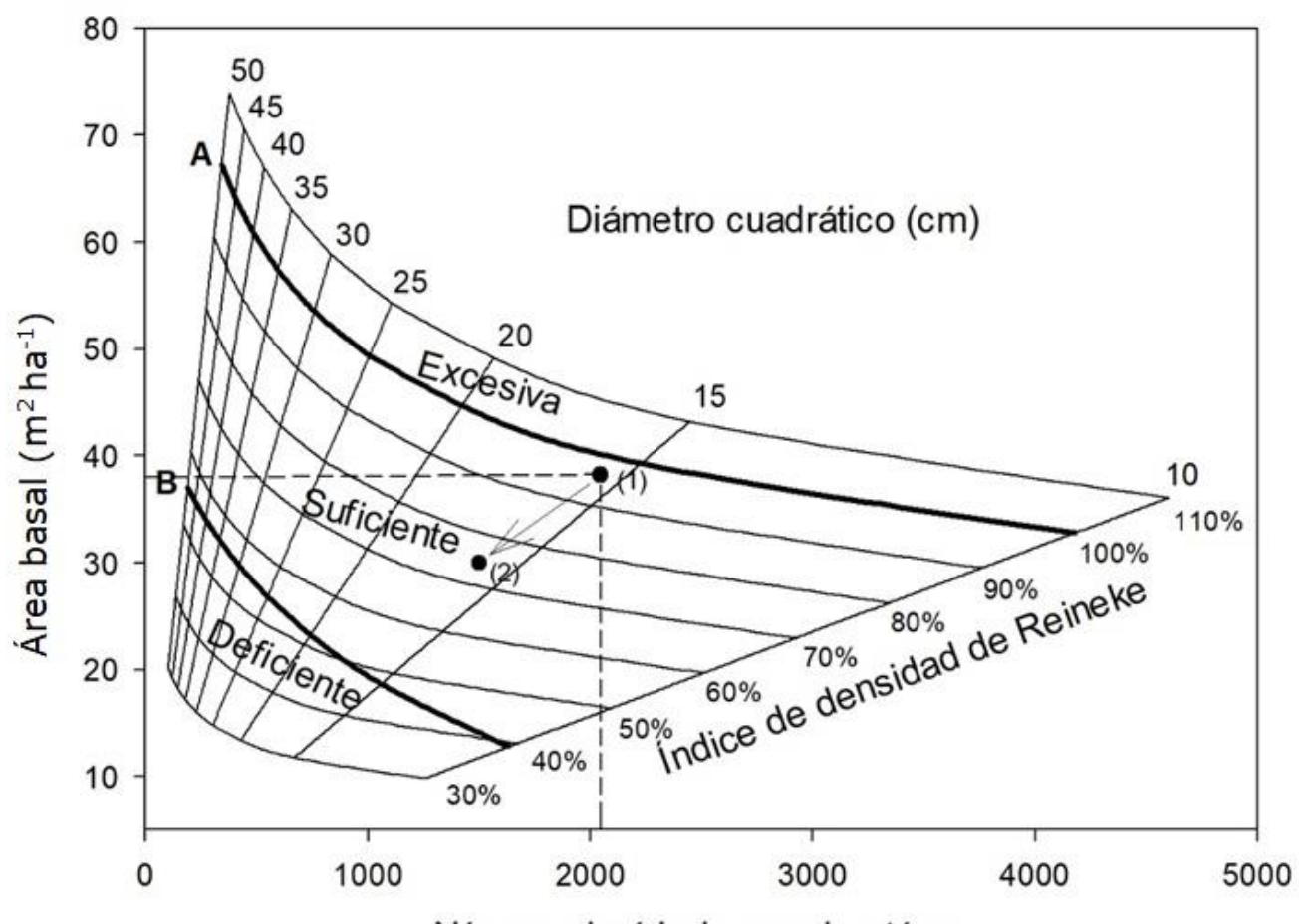

Número de árboles por hectárea

Figura 4. Guía de densidad para Pinus rudis Endl. 


\section{Conclusiones}

Los modelos ajustados para la estimación del IDR y FCC presentan buena calidad de ajuste mediante la técnica de Mínimos Cuadrados Ordinarios con coeficientes de determinación $\left(r^{2}\right)$ de 0.94 y 0.83 , respectivamente. La guía de densidad presentada en este estudio es una herramienta de apoyo al silvicultor, ya que le permitirá conocer los niveles de densidad absoluta y relativa del bosque, las necesidades de aclareos e intensidades de los mismos. La metodología expuesta es sencilla y fácil de aplicar en campo, además es confiable para la construcción de guías de densidad en rodales naturales y plantaciones forestales.

\section{Agradecimientos}

Al Tecnológico Nacional de México por el financiamiento otorgado para la realización del presente proyecto con Clave: 7801.20-PD. A las autoridades agrarias de San Miguel El Grande por las facilidades brindadas para la toma de datos de campo.

\section{Conflictos de intereses}

Los autores de este trabajo de investigación declaran no tener conflicto de intereses.

\section{Contribución por autor}

Daniel Martínez Santiago: planeación de actividades, levantamiento de información de campo, análisis de datos y elaboración del manuscrito; Prudencia Caballero Cruz y Eduardo Filio Hernández: levantamiento de información de campo y revisión del manuscrito; Alejandro Garzón Trinidad, Rosalino Ortiz Barrios, Octavio Lemuel Cruz Santiago, Ithaiz Aparicio Cuevas, y Carmela Sandoval García: levantamiento de información de campo. 


\section{Referencias}

Aguilar L., J. M. E. 2018. Estructura y diversidad de la vegetación arbórea de un bosque de galería en el estado de Puebla. Revista Mexicana de Ciencias Forestales 9(47): 230-252. Doi: 10.29298/rmcf.v9i47.154.

Álvarez T., M. F., R. Marqués G., A. Fernández M. y F. Castedo V. 2004. Influencia de la competencia en el crecimiento en sección de Pinus radiata D. Don en el bierzo (León). Sociedad Española de Ciencias Forestales 18: 129.133.

https://www.researchgate.net/publication/40836564 (1 de octubre de 2020).

Arias A., D. 2005. Morfometría del árbol en plantaciones forestales tropicales. Kurú 2(5): 19-32. https://dialnet.unirioja.es/servlet/articulo?codigo $=5123399$ (30 de septiembre de 2020).

Caballero C., P., W. Santiago J., D. Martínez S., O. L. Cruz S., E. R. Pérez S. y O. A. Aguirre C. 2018. Combustibles forestales y susceptibilidad a incendios de un bosque templado en la Mixteca Alta, Oaxaca, México. Foresta Veracruzana 20 (1): 1-9. http://www.redalyc.org/articulo.oa?id=49757295003 (30 de septiembre de 2020).

Chisman, H. H. and F. X. Schumacher. 1940. On the tree-area ratio certain of its aplications. Journal of Forestry 38 (4): 311-317. Doi: 10.1093/jof/38.4.311.

Comeau, P. G., M. While, G. Kerr and S. Hale. 2010. Maximum density-size relationships for Sitka spruce and coastal Douglas-fir in Britain and Canada. Forestry 83: 461-468. Doi: 10.1093/forestry/cpq028.

Curtis, R. O. 1970. Stand density measures: an interpretation. Forest Science 16 (4):403-414. Doi: 10.1093/forestscience/16.4.403.

Daniel, T. W., J. A. Helms y F. S Baker. 1982. Principios de Silvicultura. Segunda edición. (Traducción de Ramón Elizondo Mata, UNAM). Editorial McGraw-Hill. México, D. F., México. 482 p. 
García, E. 1998. Climas, escala 1:1000000. Comisión Nacional para el Conocimiento y Uso de la Biodiversidad. México. http://www.conabio.gob.mx/informacion/gis/ (26 de julio del 2020).

García C., X., C. Parraguirre L. y H. Ramírez M. 1996. Guía de densidad para manejo de plantaciones de Swietenia macrophylla King. (Caoba). Ciencia Forestal en México 21(80): 79-95.

Gezan, S. A., A. Ortega y E. Andenmatten. 2007. Diagramas de manejo de densidad para renovales de roble, raulí y coigüe en Chile. Bosque 28(2): 97-105.

Doi: $10.4067 /$ S0717-92002007000200002.

Gingrich, S. F. 1967. Measuring and evaluating stocking and stand density in upland hardwood forests in the Central States. Forest Science 13(1):38-53.

Doi: 10.1093/forestscience/13.1.38.

Hernández R., J., J. J. García M., H. J. Muñoz F., X. García C., T. Sáenz R., C. Flores L. y A. Hernández R. 2013. Guía de densidad para manejo de bosques naturales de Pinus teocote Schlecht. et Cham. en Hidalgo. Revista Mexicana de Ciencias Forestales 4(19): 62-77. Doi: 10.29298/rmcf.v4i19.379.

Instituto Nacional de Estadística Geografía (INEGI). 2014. Conjunto de datos vectoriales edafológico. Escala 1:1000000. Serie II. México, D.F., México. s/p. http://www.conabio.gob.mx/informacion/gis/ (26 de julio del 2020).

Instituto Nacional de Estadística Geografía (INEGI). 2016. Conjunto de datos vectoriales de la carta de Uso del suelo y vegetación. Escala 1:250000. Serie VI. México. http://www.conabio.gob.mx/informacion/gis/ (26 de julio del 2020).

Krajicek, J. E., K. A. Brinkman and S. F. Gingrich. 1961. Crown competition, a measure of density. Forest Science 7(1):35-42. Doi: 10.1093/forestscience/7.1.35. 
Lee, D. and J. Choi. 2019. Evaluating maximum stand density and size-density relationships based on the Competition Density Rule in Korean pines and Japanese larch. Forest Ecology and Management 446 (2019): 204-213.

Doi: $10.1016 /$ j.foreco.2019.05.017.

Márquez L., M. A. y R. Álvarez Z. 1995. Construcción de una guía de densidad para Pinus cooperi var. ornelasi con base en la clase de copa en Durango, México. Madera y Bosques 1(2): 23-36. Doi: 10.21829/myb.1995.121396.

Martínez L., O. 2009. Programa de Manejo Forestal Nivel Avanzado para el predio de San Miguel el Grande (2009-2018). Secretaría de Medio Ambiente y Recursos Naturales. Oaxaca, Oax., México. 101 p.

Müller U., B., R. Rodríguez y P. Gajardo. 2013. Desarrollo de una guía de manejo de la densidad en bosques de segundo crecimiento de roble (Nothofagus obliqua) en la región del Biobío. Bosque 34(2): 201-209. Doi:10.4067/S071792002013000200009.

Navarro C., C., M. A. Herrera, F. Drake A. y P. J. Donoso. 2011. Diagrama de manejo de densidad y su aplicación a raleo en bosques de segundo crecimiento de Drimys winteri en el sur de Chile. 32 (2): 175-186. Doi: 10.4067/S071792002011000200008.

Perry, J. P. Jr. 1991. The pines of Mexico and Central America. Timber Press Inc. Portland, OR, USA. 231 p.

Philbrook, J. S., J. P. Barrett and W. B. Leak. 1973. A stocking guide for eastern white pine. Research Note NE-168. Department of Agriculture, Forest Service, Northeastern. Upper Darby, PA, USA. 3 p.

Pretzsch, H. and P. Biber. 2005. A Re-Evaluation of Reineke's Rule and Stand Density Index. Forest Science 51(4): 304-320. Doi:10.1093/forestscience/51.4.304. 
Prodan, M. 1968. Forest Biometric. Pergamon Press. Oxford, UK. 447 p.

Quiñonez B., G., J. C. Tamarit U., M. Martínez S., X. García C., H. M. De Los Santos. P. and W. Santiago G. 2017. Maximum density and density management diagram for mixedspecies forests in Durango, Mexico. Revista Chapingo Serie Ciencias Forestales y del Ambiente. 24(1): 74-90. Doi: 10.5154/r.rchscfa.2017.09.056.

Ramos R., J. C., E. J. Treviño G., E. Buendía R., O. A. Aguirre C. y J. I. López M. 2017. Productividad y estructura vertical de un bosque templado con incidencia de incendios forestales. Revista Mexicana de Ciencias Forestales 8(43): 64-88. Doi: 10.29298/rmcf.v8i43.66.

Reineke, L. H. 1933. Perfecting a stand-density index for even-aged forests. Journal of Agricultural Research 46(7): 627-638.

https://naldc.nal.usda.gov/download/IND43968212/PDF (25 de marzo de 2020).

Rodríguez L., R., R. Razo Z., D. Díaz H. y J. Meza R. 2009. Guía de densidad para Pinus montezumae en su área de distribución natural en el estado de Hidalgo. Universidad Autónoma del Estado De Hidalgo. Instituto de Ciencias Agropecuarias. Pachuca, Hgo., México. 33 p.

Santiago G., W., H. M. De Los Santos P., J. R. Ángeles P., D. H. Del Valle P. y J. J. Corral R. 2013. Auto-aclareo y guías de densidad para Pinus patula mediante el enfoque de regresión de frontera estocástica. Agrociencia. 47(1) 75-89. http://www.scielo.org.mx/pdf/agro/v47n1/v47n1a7.pdf (16 de marzo de 2020).

Statsoft Inc., 2011. Statistica (data analysis software system). Version 10. www.statsoft.com (29 de enero de 2020).

Tamarit U., J. C., G. Quiñonez B., H. M. De Los Santos P., A. Castañeda M. y W. Santiago G. 2018. Diagrama para manejo de la densidad en rodales de Pinus patula Schiede ex Schltdl. \& Cham. en Puebla, México. Revista Mexicana de Ciencias Forestales 10(51): 157-181. Doi: 10.29298/rmcf.v10i51.223. 
Tamarit U., J. C., G. Quiñonez B. y J. Hernández R. 2020. Aspectos metodológicos para generar diagramas de manejo de la densidad de rodales con base en el índice de Reineke. Revista Mexicana de Ciencias Forestales 11(61): 4-26.

Doi: $10.29298 /$ rmcf.v11i61.728.

Torres R., J. M. y A. Velázquez M. 2000. Índice de densidad relativa para rodales coetáneos mezclados. Agrociencia 34(4): 497-507.

https://www.redalyc.org/pdf/302/30234413.pdf (25 de abril de 2020).

Torres R., J. M. y O. S. Magaña T. 2001. Evaluación de Plantaciones Forestales. Limusa. México, D. F., México. 472 p.

VanderSchaaf, C. L. and H. E. Burkhart. 2007. Comparison of methods to estimate Reineke's maximum size-density relationship species boundary line slope. Forest Science 53(3): 435-442. Doi: 10.1093/forestscience/53.3.435.

Vargas L., B. 1999. Caracterización de la productividad y estructura de Pinus hartwegii Lind. en tres gradientes altitudinales en el cerro Potosí, Galeana, Nuevo León. Tesis de maestría. Facultad de Ciencias Forestales. Universidad Autónoma de Nuevo León. Linares, N. L., México. 93 p.

http://cdigital.dgb.uanl.mx/te/1020125428.pdf (30 de septiembre de 2020).

Yoda, K., T. Kira, H. Ogawa and K. Hozumi. 1963. Self-thinning in overcrowded pure stands under cultivated and natural conditions. Journal of Biology 14: 107-129.

Zepeda B., E. M. y M. E. Villareal D. 1987. Guía de densidad para Pinus hardwegii Lind., Zoquiapan, México. Universidad Autónoma de Chapingo, División de Ciencias Forestales. Texcoco, Edo. de Méx., México. 52 p.

\section{(c) (1) (9)}

Todos los textos publicados por la Revista Mexicana de Ciencias Forestales -sin excepciónse distribuyen amparados bajo la licencia Creative Commons 4.0 Atribución-No Comercial (CC BY-NC 4.0 Internacional), que permite a terceros utilizar lo publicado siempre que mencionen la autoría del trabajo y a la primera publicación en esta revista. 